\title{
MANAGEMENT OF THE AXILLA IN PATIENTS WITH BREAST CANCER AND ONE OUT OF ONE POSITIVE SENTINEL LYMPH NODE. CAN WE OMIT AXILLARY LYMPH NODE CLEARANCE?
}

Foivos Irakleidis', Ashutosh Tondare ${ }^{1}$, Hisham Hamed ${ }^{1}$, Ashutosh Kothari

'Guy's and St Thomas' NHS Foundation Trust, Breast Surgery Unit - London, United Kingdom.

Background: Over the past three decades, the treatment of the axilla in breast cancer management continues to change. Current treatment strategies aim to achieve regional nodal control associated with reduced incidence of lymphedema and other long-term complications. In this study, we analyzed our tertiary center's database of patients who had a single retrieved sentinel node (SN) that was positive for macrometastatic disease. We focused on AMAROS trial outcomes and the future view of treating this cohort of patients with axillary radiotherapy (RT) instead of axillary node clearance (ANC). Methods: Both the literature review and the 5-year retrospective analysis of our database were performed, focusing on the management of the axilla in patients with breast cancer with one-in-one positive SN. Results: A total of 24 patients who had surgery as primary treatment had one-in-one positive SN. All patients had the clinical and radiological assessment of their axilla prior to their sentinel lymph node biopsy (SNB). In all, $92 \%$ of these patients had a complete ANC, $50 \%$ of them had zero additional positive nodes, $21 \%$ had only one additional positive node, and a further $21 \%$ had more than one additional positive node. One patient was planned for ANC but died from chemotherapy-related complications and one more patient had alternative axillary RT instead of ANC. Of note, $80 \%$ of patients who had three or more positive axillary lymph nodes following ANC had indeed evidence of advanced locoregional disease and thus would not be eligible for alternative axillary RT, as compared with one patient who had a multifocal disease, could have axillary RT but had a heavy axillary burden on ANC. Finally, $71 \%$ of patients could have been offered alternative axillary RT but had ANC instead. Fourteen patients from this group had chest wall and supraclavicular fossa RT after their initial surgery, and thus, the addition of axillary RT instead of ANC could have been offered. Conclusion: In patients with early breast cancer and clinically node-negative axilla, disease burden in non-SN is limited and ANC may entail overtreatment. In view of low recurrence and complication rates seen in the AMAROS trial, axillary irradiation appears to be a valid and safe alternative when compared with ANC in patients with one-in-one positive SN.

Keywords: Axillary Radiotherapy; Sentinel Lymph Node; Axillary Lymph Node Clearance. 